\title{
Early Intervention
} IN PSYCHIATRY

\section{Development and pilot testing of an online monitoring tool of depression symptoms and side effects for young people being treated for depression}

\begin{tabular}{|r|l|}
\hline Journal: & Early Intervention in Psychiatry \\
\hline Manuscript ID: & EIP-2013-105.R1 \\
\hline Danuscript Type: & Brief Report \\
\hline Complete List of Authors: & $\begin{array}{l}\text { Hetrick, Sarah; University of Melbourne, Orygen Youth Health Research } \\
\text { Centre, Centre for Youth Mental Health; University of Melbourne, } \\
\text { headspace Centre of Excellence, Centre for Youth Mental Health } \\
\text { Dellosa, Maria; eheadspace, } \\
\text { Simmons, Magenta; The University of Melbourne, Centre for Youth Mental } \\
\text { Health; University of Melbourne, headspace Centre of Excellence, Centre } \\
\text { for Youth Mental Health } \\
\text { Philips, Lisa; University of Melbourne, School of Psychological Sciences }\end{array}$ \\
\hline Keywords: & $\begin{array}{l}\text { Depression, Evidence based practice, monitoring, suicide related } \\
\text { behaviours, youth }\end{array}$ \\
\hline \multicolumn{2}{|c}{} \\
\hline
\end{tabular}




\section{Development and pilot testing of an online monitoring tool of depression symptoms and side effects for young people being treated for depression}

Dr Sarah E Hetrick*, Senior Research Fellow, headspace Centre for Excellence in Youth Mental Health, Orygen Youth Health Research Centre, Centre for Youth Mental Health, The University of Melbourne, Locked Bag 10, Parkville, Victoria 3052.

Ms Maria Kristina Dellosa, School of Psychological Sciences, The University of Melbourne, Victoria $3010^{1}$

Dr Magenta Simmons, Research Fellow, headspace Centre for Excellence in Youth Mental Health, Orygen Youth Health Research Centre, Centre for Youth Mental Health, The University of Melbourne, Locked Bag 10, Parkville, Victoria 3052.

Dr Lisa Phillips, Associate Professor, Department of Psychology, The University of Melbourne, Victoria 3010

*Corresponding author:

Dr Sarah Hetrick, Orygen Youth Health Research Centre, Locked Bag 10, Parkville, Victoria, Australia, 3052. Phone: +61 3 93422800. Fax: +61 39027 0199. Email: shetrick@unimelb.edu.au

Keywords

Depression, evidence based practice, monitoring, suicide related behaviours, youth

\footnotetext{
${ }^{1}$ The study was undertaken while Ms Dellosa was a student at the University of Melbourne; her current address is eheadspace, headspace National Youth Mental Health Foundation, Level 3, South Tower, 485 La Trobe Street, Melbourne 3000
} 


\begin{abstract}
Aim

To develop and examine the feasibility of an online monitoring tool of depressive symptoms, suicidality and side effects.

\section{Methods}

The online tool was developed based on guideline recommendations and employed already validated and widely used measures. Quantitative data about its use, and qualitative information on its functionality and usefulness were collected from surveys, a focus group and individual interviews.

Results

Fifteen young people completed the tool between one and 12 times and reported it was easy to use. Clinicians suggested it was too long and could be completed in the waiting room to lessen impact on session time. Overall, clients and clinicians who used the tool found it useful.

\section{Conclusions}

Results show that an online monitoring tool is potentially useful as a systematic means for monitoring symptoms, but further research is needed including how to embed the tool within clinical practice.
\end{abstract}




\section{Introduction}

Guidelines assist clinicians to provide evidence based and effective interventions for young people with depression (5-9). They consistently recommend regular monitoring of symptoms and, for those prescribed an antidepressant, of adverse effects and suicide risk given this risk is increased on antidepressants (10-12). However, research has shown these recommendations are not uniformly implemented $(13,14)$. Based on a study of barriers to monitoring (15), a self-report tool was designed to assist clinicians to undertake adequate monitoring.

The aim of the current study was to examine the feasibility of this tool.

\section{Methods}

\section{Setting}

The Youth Mood Clinic (YMC) is part of Orygen Youth Health (OYH), a public youth mental health service for those aged $15-24$ living in the northwestern metropolitan area of Melbourne, Australia. Eighty-five percent of YMC clients have major depressive disorder; $80 \%$ receive mediation given the severity of disorder, established by the Patient Health Questionnaire (PHQ-9; (16)) and clinical assessment. Psychiatrists regularly review clients, and case managers (clinical psychologists'; social workers; occupational therapists) deliver weekly CBT and case management.

\section{Participants}


New or already established YMC clients and all clinicians in the YMC were invited to participate.

Design and development of the monitoring tool

The tool was based on guideline recommendations for the regular monitoring of symptoms and side effects, particularly suicidality.

\section{Phase 1: Development}

The PHQ-9 (16) was chosen to monitor depression symptoms given its use in the YMC . It has sound psychometric properties (16) and is used in those as young as 12(17). While mostly used for screening, it is a useful outcome measure of depression (17-19)..

The Suicidal Ideation Questionnaire-Junior (SIQ-JR;(20)) was chosen to monitor suicidal ideation on the basis of a systematic review in which it was the most common tool (21). It has sound psychometric properties (20) and is used in those aged as young as $11(20,22)$.

A checklist of side effects was constructed using the Antidepressant Side-Effect Checklist (ASEC) and a synthesis of reported side effects from a systematic review of antidepressants in adolescents (23).

The tool was designed to be available online; at the YMC it was available via an PAD.

Phase 2: Refinement 
The Platform Team at OYH, comprised of past and present clients, participated in a focus group. Feedback included: (i) a preference for radio buttons rather than dropdown options and (ii) a preference to exclude images.

Feedback from YMC clinicians included using language consistent with the service (e.g. "case manager" rather than "clinician") and limiting free text boxes given concerns these would be used to communicate distress outside of clinic hours with the expectation of an immediate response.

\section{Procedure}

A researcher contacted the YMC administrator regularly to obtain information about new referrals. Clients were contacted by telephone, information about the study provided and verbal consent obtained. If the client was under 18 years of age, verbal consent was also obtained from a parent or guardian. Signed written consent was obtained at their next appointment. Written parental consent was obtained during this appointment or via post. Written consent was obtained from clinicians during the consultation session with YMC.

Participants were asked to complete the tool once a week for up to three months. Clients could fill in the tool at any location with Internet access at any time with the exception suicidal ideation items. These were completed at the beginning of their regular treatment session with their clinician on an iPad. Once completed the client handed the iPad to their clinician, with the iPad programmed to display SIQ-JR responses with an indication regarding the meaning of the scores (i.e. SIQ-JR score of $>31$ or a score of $>4$ 
on items $2,3,4,7,8,9,13$ or 18$)$.. Clinicians could then respond immediately according to the YMC protocols for suicidal risk.

Clinicians had no access to raw data of clients PHQ-9 and SIQ-JR scores but received a chart of these scores after 4 weeks and could choose to share this with their client. They received an email regarding side effects that were endorsed, which they could report to the treating psychiatrist for immediate action.

Clients and clinicians also filled in a survey about the functionality and usefulness of the tool. A focus group with YMC clinician's and telephone interviews with clients were conducted to further investigate these issues.

\section{Ethics}

This study received ethics approval from the Melbourne Health Research and Ethics Committee (reference number 2010.240).

Data Analysis

Frequency data and descriptive statistics were used to report on use of the tool. Analysis of qualitative data was driven by the research aims regarding functionality and usefulness and was undertaken in accordance with Braun and Clarke's methods (24).

\section{Results}

Quantitative Data 


\begin{abstract}
All seven YMC case managers and 34 of their clients consented to participate.. Doctors were not involved as they see clients less frequently and were difficult to liaise with given case load and time constraints.
\end{abstract}

Of the 34 clients who provided consent, six were unable to commence because a signed parental consent form was not returned. A further 13 did not commence use of the tool. The tool was filled in by 15 clients (mean age $18.74, \mathrm{SD}=2.65$ ). The mean PHQ-9 score was 13.3 which indicates a moderate level of severity; $73 \%$ were on medication, They filled the tool in between one and 12 times $(\mathrm{M}=5.33, \mathrm{SD}=4.08$; Table 4$)$. The tool was mostly completed with the clinician (Table 1).

Table 1

Frequency and location of use

\begin{tabular}{lll} 
Location & $\underline{\text { Frequency }}$ & $\underline{\text { Percentage }(\%)}$ \\
\hline With clinician & 51 & 62.50 \\
At home & 25 & 28.75 \\
Waiting room at clinic & 5 & 6.25 \\
Library & 2 & 1.25 \\
School & 1 & 1.25 \\
Total & 80 & 100 \\
\hline
\end{tabular}

Qualitative Data

Seven clients and seven clinician completed surveys, and six interviews with clients plus a focus group with clinicians (7) were undertaken. 
Functionality. The time taken to complete the tool was acceptable to most clients (range 2 to 10 minutes). One client reported that it took too long to complete.

All but one client reported the online format was "good", stating it was easier than having a "conversation with the case manager", and that it was "simple" to complete. One client found it "a bit weird" because they were not used to it and two reported it was not capable of "capturing what you felt by ticking a box".

Clinicians all reported liking the tool but were concerned about its length and that too much "time [was] taken in session". Most clinicians suggested implementing the tool in the waiting room.

Usefulness. Most clients stated the tool helped them in some way. Generally it helped them to (i) understand more about their symptoms, (ii) understand how symptoms fluctuated in response to life events, and (iii) allowed them to feel more in control of managing symptoms. Two clients stated the tool helped them change their behaviour of "not eating and sleeping". Only one client said that the tool was not helpful because "it wasn't telling me anything I didn't (already) know”.

No clinician showed their client graphs of their PHQ-9 or SIQ-JR scores, however, clients generally said this would have been helpful.

Clinicians commented that the tool helped them understand more about their client's risk. One clinician said that it was useful for informing treatment planning and another 
found it useful for engagement "particularly with clients who are reluctant to disclose information to clinicians".

\section{Discussion}

Undertaking systematic monitoring of symptoms and side effects, using an online format can be beneficial. Clients and clinicians had mostly positive feedback about the functionality and usefulness of the tool. Time taken to complete the tool was generally acceptable and clients reported it easy to use. Clients identified a particular benefit as having a greater understanding of and control over symptoms, consistent with the selfmanagement literature (25). It was therefore surprising that clinicians did not share with their clients PHQ or SIQ scores; particularly given clients thought this would have been useful.

Clinicians reported understanding more about their client's risk via the tool. It was helpful for engaging clients, particularly those who were reluctant to disclose information, suggesting self-monitoring can foster a more collaborative relationship between clinician and clients. Given previous research (25) and feedback from clients, this collaboration may be enhanced by the careful sharing of symptom scores over the course of treatment.

However, the uptake of the tool was relatively poor indicating that further work is needed to embed the tool within clinical practice. The setting, protocols and clinical management tools already in use need to be considered as also highlighted in primary care research we have undertaken (26). Further suggestions from clinicians were 
consistent with previous research (27) and included reducing the length of the tool and having it completed in the waiting room. These improvements, implementation tailored to individual settings, as well as providing evidence of utility in terms of improving clinical practice via weekly feedback of scores (as demonstrated in previous research (28)) would improve the likelihood that the tool would improve adherence to guideline recommendations about monitoring. Further testing of the tool in this regard is required.

No doctors provided feedback and surveys and phone interviews were not completed by all clients who used the tool, so generalisability may be limited; investigating how doctors, younger clients and clients being treated in different settings use the tool is important for future studies.

\section{Acknowledgements}

Our thanks to Mr Greg Wadley who did the programming and web design. SH is on a National Health and Medical Research Council Early Career Fellowship. OYHRC is affiliated with Melbourne Health and the University of Melbourne and receives funding from the Colonial Foundation. 


\section{References}

1. Costello JE, Erkanli A, Angold A. Is there an epidemic of child or adolescent depression? Journal of Child Psychology and Psychiatry. 2006;47(12):1263-71.

2. Lewinsohn PM, Rohde P, Seeley JR. Major depressive disorder in older adolescents: Prevalence, risk factors and clinical implications. Clin Psychol Rev. 1998;18(7):765-94.

3. Birmaher B, Ryan ND, Williamson DE, Brent DA, Kaufman J, Dahl RE, et al. Childhood and adolescent depression: A review of the past 10 years: Part 1. J Am Acad Child Adolesc Psychiatry. 1996;35(11):1427-39.

4. Gould MS, King R, Greenwald S, Fisher P, Schwab-Stone M, Kramer R, et al. Psychopathology associated with suicidal ideation and attempts among children and adolescents. J Am Acad Child Adolesc Psychiatry. 1998;37(9):915-23.

5. American Academy of Child and Adolescent Psychiatry (AACAP). Practice parameters for the assessment and treatment of children and adolescents with depressive disorders. J Am Acad Child Adolesc Psychiatry. 2007;46(11):1503-26.

6. Cheung AH, Zuckerbrot RA, Jensen PS, Ghalib K, Laraque D, Stein REK, et al. Guidelines for adolescent depression in primary care (GLAD-PC): II treatment and ongoing management. Pediatrics. 2007;120:e1313-26.

7. National Institute for Health and Clinical Excellence NICE. Depression in Children and Young People: Identification and management in primary, community and secondary care. Leicester, UK: The British Psychological Society; 2005.

8. Zuckerbrot RA, Cheung AH, Jensen PS, Stein REK, Laraque D, and the GLADPC Steering Group. Guidelines for adolescent depression in primary care (GLAD-PC): I identificiation, assessment and initial management. Pediatrics. 2007;120:e1313-26.

9. McDermott B, Baigent M, Chanen A, Fraser L, Graetz B, Hayman N, et al. beyondblue Expert Working Committee (2010) Clinical practice guidelines: Depression in adolescents and young adults. Melbourne: beyondblue: the national depression initiative; 2011.

10. Hetrick SE, McKenzie JE, Cox GE, Simmons MB, Merry SN. Newer generation antidepressants for depressive disorders in chidlren and adolescents. Cochrane Database of Systematic Reviews. 2012; Issue 12.

11. Dubicka B, Hadley S, Roberts C. Suicidal behaviour in youths with depression treated with new-generation antidepressants: meta-analysis. Br J Psychiatry. 2006;189:393-8.

12. Hammad TA, Laugren T, Racoosin J. Suicidality in pediatric patients treated with antidepressant drugs. Arch Gen Psychiatry. 2006;63:332-9.

13. Perera A, Gupta P, Samuel R, Berg B. A survey of anti-depressant prescribing practice and the provision of psychological therapies in a South London CAMHS from 2003-2006. Child and Adolescent Mental Health. 2007;12(2):70-2.

14. Hetrick SE, Thompson A, Yuen K, Finch S, Parker AG. Is there a gap between recommended and 'real world' practice in the management of depression in young people? A medical file audit of practice. BMC Health Services. 2012;12:178.

15. Hetrick SE, Simmons M, Thompson A, Parker AG. What are specialist mental health clinician attitudes to guideline recommendations for the treatment of depression in young people? Aust N Z J Psychiatry. 2011;45(11):993-1001. 
16. Spitzer R, Kroenke K, Williams J. Validation and utility of a self-report version of PRIME-MD: the PHQ Primary Care Study. Journal of the American Medical Association. 1999;282:1737-44.

17. Richardson L, McCauley E, Katon W. Collaborative care for adolescent depression: A pilot study. Gen Hosp Psychiatry. 2008;31:36-45.

18. Chen TM, Huang FY, Chang C, Chung H. Using the PHQ-9 for depression screening and treatment monitoring for Chinese Americans in primary care. Psychiatr Serv. 2006;57:976-81.

19. Patten SB, Schopflocher D. Longitudinal epidemiology of major depression as assessed by the Brief Patient Health Questionnaire (PHQ-9). Compr Psychiatry. 2009;50:26-33.

20. Reynolds WM. Suicidal Ideation Questionnaire (SIQ): Professional Manual. Odessa, FL: Psycholgogical Assessment Resources; 1987.

21. Robinson J, Hetrick SE, Martin C. Preventing suicide in young people: systematic review Australian New Zealand Journal of Psychiatry. 2011;45:3-26.

22. Reynolds WM, Mazza JJ. Assessment of suicidal ideation in inner-city children and young adolescents: reliability and validity of the Suicidal Ideation QuestionniareJR. School Psychology Review. 1999;28(1):17-30.

23. Hetrick SE, Merry S, McKenzie J, Sindahl P, Proctor M. Selective serotonin reuptake inhibitors (SSRIs) for depressive disorders in children and adolescents (Review). Cochrane Database of Systematic Reviews In: The Cochrane Library 2007; Issue 3(Art. No.: CD004851. DOI: 10.1002/14651858).

24. Braun V, Clarke V. Using thematic analysis in psychology. Qualitative Research in Psychology. 2006;3:77-101.

25. Barlow J, Wright C, Sheasby J, Turner A, Hainsworth J. Self-management approaches for people with chronic conditions: A review. Patient Education and Counselling. 2002;48(2):177-87.

26. Hetrick SE, Simmons MB, Sanci L, Gunn J. Monitoring depressive symptoms and suicidality in young people in primary care settings: focus groups with general practitioners. Aust Fam Physician. 2013; In press (accepted 21 November).

27. Timimi S, Tetley D, Burgoine W, Walker G. Outcome Orientated Child and Adolescent Mental Health Services (OO-CAMHS): a whole service model. Clin Child Psychol Psychiatry. 2013;18(2):169-84. Epub 2012/05/03.

28. Timimi S, Tetley D, Burgoine W, Walker G. Outcome Orientated Child and Adolescent Mental Health Services (OO-CAMHS): a whole service model. Clinical Child Psycholology and Psychiatry. 2013;18(2):164-84. 


\section{Development and evaluation-pilot testing of an online monitoring tool of} depression symptoms and side effects for young people being treated for depression

Dr Sarah E Hetrick*, Senior Research Fellow, headspace Centre for Excellence in Youth Mental Health, Orygen Youth Health Research Centre, Centre for Youth Mental Health, The University of Melbourne, Locked Bag 10, Parkville, Victoria 3052.

Ms Maria Kristina Dellosa, School of Psychological Sciences, The University of Melbourne, Victoria $3010^{1}$

Dr Magenta Simmons, Research Fellow, headspace Centre for Excellence in Youth Mental Health, Orygen Youth Health Research Centre, Centre for Youth Mental Health, The University of Melbourne, Locked Bag 10, Parkville, Victoria 3052.

| Dr Lisa Phillips, Associate Professor, Department of Psychology, The University of Melbourne, Victoria 3010

*Corresponding author:

Dr Sarah Hetrick, Orygen Youth Health Research Centre, Locked Bag 10, Parkville, Victoria, Australia, 3052. Phone: +61 3 93422800. Fax: +61 39027 0199. Email: shetrick@unimelb.edu.au

Keywords

Depression, evidence based practice, monitoring, suicide related behaviours, youth

\footnotetext{
${ }^{1}$ The study was undertaken while Ms Dellosa was a student at the University of Melbourne; her current address is eheadspace, headspace National Youth Mental Health Foundation, Level 3, South Tower, 485 La Trobe Street, Melbourne 3000
} 


\section{Conclusions}

Results show that an online monitoring tool could is potentially be useful as a systematic means for monitoring symptoms, but further research is needed including how to work is needed to-embed the tool within clinical practice. 


\title{
Introduction
}

\begin{abstract}
The prevalence of depression in young people is high $($ e.g. 1,2$)$ and the potential negative impacts on developing and maintaining good relationships, vocational attainment and achievement are significant $(2,3)$. There is a high risk of self harm and suicide (4). Therefore, it is critical that depression is effectively treated.
\end{abstract}

Guidelines assist clinicians to provide evidence based and effective interventions for young people with depression (5-9). Guidelines-They consistently recommend regular monitoring of symptoms and, for those prescribed an antidepressant, regular monitoring of adverse effects and suicide related behaviour-suicide risk given this risk is increased one known risks of these behaviours for young people on antidepressants (10-12).

However, research has shown that such monitoring isthese recommendations are not uniformly implemented $(13,14)$. Based on a study of barriers to monitoring (15), including a perceived lack of time and expertise, a self-report tool was designed with the view to assisting clinicians to ensure-undertake adequate monitoring by the regular collection of standardised information.

The aim of the current study was to examine the feasibility_, functionality and usefulness of this tool.

\section{Methods}

Setting 
The Youth Mood Clinic (YMC) is part of Orygen Youth Health $(\mathrm{OYH})_{2}$ - is a public youth mental health service for those aged $15-24$ living in the northwestern metropolitan area of Melbourne, Australia. Eighty-five percent of YMC clients have major depressive disorder; $80 \%$ receive mediation given the severity of disorder, established by the Patient Health Questionnaire (PHQ-9; (16)) and clinical assessment. $\underline{\text { Psychiatrists regularly review clients, and case managers (clinical psychologists'; }}$ $\underline{\text { social workers; occupational therapists) deliver weekly CBT and case }}$ management.

\section{Participants}

$\underline{\text { New or already established lients of } \underline{Y M C} \text { clients and }}$. In addition, all clinicians in the YMC were invited to participate.

Design and development of the monitoring tool

The content of the tool was based on guideline recommendations for the regular monitoring of that state that symptoms and side effects, particularly suicidality, be assessed regularly.

\section{Phase 1: Development}

The Patient Health QuestionnairePHQ-9-(PHQ 9; (16)) was chosen to monitor depression symptoms given its use in the as the-YMC already used it. It has sound psychometric properties (16) and is used in those as young as 12(17). While mostly used for screening, it is alse-a useful outcome measure of depression (17-19).-Clients 
were also given a free-text box to provide details of events that may have influenced their mood.

The Suicidal Ideation Questionnaire-Junior (SIQ-JR;(20)) was chosen to monitor suicidal ideation on the basis of a systematic review in which it was the most eommenly common used-tool (21). It has sound psychometric properties (20) and is used in those aged as young as $11(20,22)$.

A checklist of side effects was constructed on the basis ofusing the Antidepressant SideEffect Checklist (ASEC) and a synthesis of reported side effects in trials included infrom a systematic review of selective serotonin reuptake inhibitors (SSRIs)antidepressants in adolescents (23).

The tool was designed to be available online; at the YMC it was available via an PAD.

\section{Phase 2: Refinement}

The Platform Team at OYH, comprised of past $\theta$ rand present clients, participated in a focus group. Feedback included: (i) a preference for grids of radio buttons rather than dropdown options and (ii) a preference to exclude images from the tool.

Feedback from consultation with-YMC clinicians included using language that was consistent with the service (e.g. "case manager" rather than "clinician") and limiting free text boxes given concerns these would be used to communicate distress outside of clinic hours with the expectation of an immediate response. 


\section{Procedure}

A researcher contacted the YMC administrator regularly to obtain information about new referrals. Current and new $\underline{C}$ elients were contacted by telephone, information about the study provided and verbal consent obtained. If the client was under 18 years of age, verbal consent was also obtained via telephone-from a parent or guardian. Signed written consent was obtained at their next appointment. Written parental consent was obtained either-during this appointment or via post. Written consent had beenwas obtained from clinicians during the consultation session with YMC.

Participants were asked to complete the tool once a week for up to three months. Clients had the option tocould fill in the tool at any location with Internet access at any time with the exception of items about suicidal ideation items. These, which were completed at the beginning of their regular treatment session with their clinician -on an iPad. Once completed the client handed the iPad to their clinician, with the iPad programmed to display SIQ-JR responses with an indication regarding the meaning of the scores (i.e.

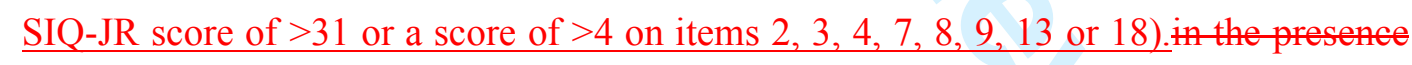
of their clinician who received immediate feedback regarding risk. Clinicians could then $\underline{\text { respond immediately according to the YMC protocols for suicidal risk. }}$

Clinicians had no access to raw data ofreceived a chart of their clients PHQ-9 and SIQJR scores but received a chart of these scores after 4 weeks and could choose to share this-graph with their client. They received an email regarding side effects that were endorsed, which they could report to the treating psychiatrist for immediate action. 


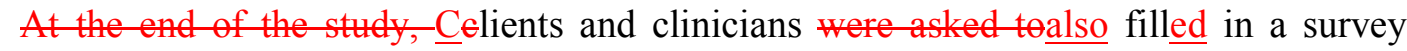
about the functionality and usefulness of the tool. A focus group with YMC clinician's and telephone interviews with clients were conducted to further investigate these issues.

\section{Ethics}

This study received ethics approval from the Melbourne Health Research and Ethics Committee (reference number 2010.240).

\section{Data Analysis}

Frequency data and descriptive statistics were used to report on elients'-use of the tool. Analysis of qualitative data was driven by the research aims regarding functionality and usefulness- and was of the tool. This analysis was undertaken in accordance with Braun and Clarke's methods (24).

\section{Results}

Quantitative Data

All seven YMC case managers in the YMC, all allied health professionals-and 34 of their clients consented to participate. in the study. Doctors were not involved as they see $\underline{\text { clients less frequently and were difficult to liaise with given case load and time }}$ constraints. 
Of the 34 clients who provided consent, six clients-were unable to commence because a signed parental consent form was not returned. A further 13 did not commence use of the tool. The tool was filled in by 15 clients (mean age $18.74, \mathrm{SD}=2.65$ ). The mean PHQ-9 score was 13.3 which indicates a moderate level of severity; $73 \%$ were on medication, They filled the tool in who filled in the tool-between one and 12 times $(\mathrm{M}=$ 5.33, $\mathrm{SD}=4.08$; Table 4). The tool was mostly completed with the clinician (Table 1).

Table 1

Frequency and location of $\underline{u}$ Use in Location Options

\begin{tabular}{lll} 
Location & $\underline{\text { Frequency }}$ & $\underline{\text { Percentage }(\%)}$ \\
\hline With clinician & 51 & 62.50 \\
At home & 25 & 28.75 \\
Waiting room at clinic & 5 & 6.25 \\
Library & 2 & 1.25 \\
School & 1 & 1.25 \\
Total & 80 & 100 \\
\hline
\end{tabular}

Qualitative Data

Seven clients and seven clinician completed surveys, and six telephone-interviews with clients and-plus a focus group with clinicians (7) were undertaken.

Functionality. The time taken to complete the tool was acceptable to most clients (range 2 to 10 minutes). One client reported that it took too long to complete.

All but one client reported the online format was "good", stating it was easier than having a "conversation with the case manager", and that it was "simple" to complete. 
One client found it "a bit weird" because they were not used to it and two clients reported that some questions wereit was limiting and-not capable of "capturing what you felt by ticking a box".

Clinicians all reported liking the tool but. However, they-were concerned about its length and that too much "time [was] taken in session". Most clinicians suggested implementing the tool in the waiting room.

Usefulness. Most clients stated some way that the tool helped them in some way. Clients-Ggenerally reported-it helped them to (i) understand more about their symptoms, (ii) understand how symptoms fluctuated in response to life events, and (iii) allowed them to feel more in control of managing symptoms. Two clients stated the tool helped them to monitor their mood and-change their behaviour of "not eating and sleeping" appropriately. Only one client said that the tool was not helpful because "it wasn't telling me anything I didn’t (already) know”.

No clinician showed their client graphs of their PHQ-9 or SIQ-JR scores, however, clients generally said this would have been helpful.

Clinicians commented that the tool was useful and helped them to-understand more about their client's risk. One clinician said that the toolit was useful for informing treatment planning and another found the toolit useful for engagement "particularly with clients who are reluctant to disclose information to clinicians". 


\section{Discussion}

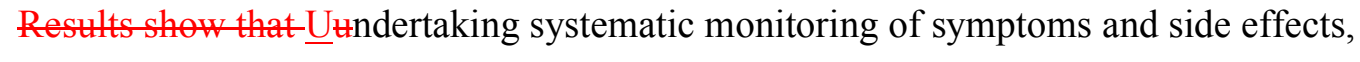
using an online format can be beneficial. Clients and clinicians had mostly positive feedback about the functionality and usefulness of the tool. This supports the use of innovative technology such as iPADs in a clinical setting.

Time taken to complete the tool was generally acceptable and clients reported finding it easy to use. Clients identified aA particular benefit as having identified by clients was having a greater understanding of and control over their-symptoms-and an increased sense of control of them ${ }_{2}$. This is consistent with the self-management literature (25). It was therefore surprising that clinicians did not share with their clients PHQ or SIQ scores; particularly given clients thought this would have been useful.

Clinicians reported that the tool helped them-understanding more about their client's risk via the tool. It was also found to be-helpful for engaging with-clients, stch asparticularly those who were reluctant to disclose information ${ }_{2}$. This feedback suggestssuggesting that self-monitoring helped tocan foster a more collaborative relationship between clinician and clients. Given previous research (25) and feedback from clients, this collaboration may be enhanced by the careful sharing of symptom scores over the course of treatment.

On the other handHowever, the uptake of the tool was relatively poor indicating that further work is needed to successfully embed the tool within clinical practice. (26) The $\underline{\text { setting, protocols and clinical management tools already in use need to be considered as }}$ 
also highlighted in primary care research we have undertaken (26). Further Useful suggestions from clinicians were consistent with previous research (27) and included reducing the length of the tool and making it available forhaving it completedion in the waiting room.. This is consistent with other recent findings (27). These improvements, $\underline{\text { implementation tailored to individual settings, as well as providing evidence of utility in }}$ $\underline{\text { terms of improving clinical practice via weekly feedback of scores (as demonstrated in }}$ previous research (28)) would improve the likelihood that the tool would improve $\underline{\text { adherence to guideline recommendations about monitoring. Further testing of the tool in }}$ this regard is required. Making the graph illustrating weekly depressive and suicidal symptom scores available to clients as well as clinicians may be useful.

The clinicians in the study were all allied health professionals and-Nno doctors provided
feedback and Further, surveys and phone interviews were not completed by all clients
who used the tool, so the generalisability may be limited; investigating how doctors,
younger clients and clients being treated in different settings use the tool is important
for future studies. however, results show the tool is functional, potentially useful and
may assist clinicians to undertake monitoring according to guideline recommendations.

\section{Acknowledgements}

Our thanks to Mr Greg Wadley who did the programming and web design. SH is on a National Health and Medical Research Council Early Career Fellowship. OYHRC is affiliated with Melbourne Health and the University of Melbourne and receives funding from the Colonial Foundation. 
1

2

3

4

5

6

7

8

9

10

11

12

13

14

15

16

17

18

19

20

21

22

23

24

25

26

27

28

29

30

31

32

33

34

35

36

37

38

39

40

41

42

43

44

45

46

47

48

49

50

51

52

53

54

55

56

57

58

59

60 


\section{References}

1. Costello JE, Erkanli A, Angold A. Is there an epidemic of child or adolescent depression? Journal of Child Psychology and Psychiatry. 2006;47(12):1263-71.

2. Lewinsohn PM, Rohde P, Seeley JR. Major depressive disorder in older adolescents: Prevalence, risk factors and clinical implications. Clin Psychol Rev. 1998;18(7):765-94.

3. Birmaher B, Ryan ND, Williamson DE, Brent DA, Kaufman J, Dahl RE, et al. Childhood and adolescent depression: A review of the past 10 years: Part 1. J Am Acad Child Adolesc Psychiatry. 1996;35(11):1427-39.

4. Gould MS, King R, Greenwald S, Fisher P, Schwab-Stone M, Kramer R, et al. Psychopathology associated with suicidal ideation and attempts among children and adolescents. J Am Acad Child Adolesc Psychiatry. 1998;37(9):915-23.

5. American Academy of Child and Adolescent Psychiatry (AACAP). Practice parameters for the assessment and treatment of children and adolescents with depressive disorders. J Am Acad Child Adolesc Psychiatry. 2007;46(11):1503-26.

6. Cheung AH, Zuckerbrot RA, Jensen PS, Ghalib K, Laraque D, Stein REK, et al. Guidelines for adolescent depression in primary care (GLAD-PC): II treatment and ongoing management. Pediatrics. 2007;120:e1313-26.

7. National Institute for Health and Clinical Excellence NICE. Depression in Children and Young People: Identification and management in primary, community and secondary care. Leicester, UK: The British Psychological Society; 2005.

8. Zuckerbrot RA, Cheung AH, Jensen PS, Stein REK, Laraque D, and the GLADPC Steering Group. Guidelines for adolescent depression in primary care (GLADPC): I identificiation, assessment and initial management. Pediatrics. 2007;120:e1313-26.

9. McDermott B, Baigent M, Chanen A, Fraser L, Graetz B, Hayman N, et al. beyondblue Expert Working Committee (2010) Clinical practice guidelines:

Depression in adolescents and young adults. Melbourne: beyondblue: the national depression initiative; 2011.

10. Hetrick SE, McKenzie JE, Cox GE, Simmons MB, Merry SN. Newer generation antidepressants for depressive disorders in chidlren and adolescents. Cochrane Database of Systematic Reviews. 2012; Issue 12.

11. Dubicka B, Hadley S, Roberts C. Suicidal behaviour in youths with depression treated with new-generation antidepressants: meta-analysis. Br J Psychiatry. 2006;189:393-8.

12. Hammad TA, Laugren T, Racoosin J. Suicidality in pediatric patients treated with antidepressant drugs. Arch Gen Psychiatry. 2006;63:332-9.

13. Perera A, Gupta P, Samuel R, Berg B. A survey of anti-depressant prescribing practice and the provision of psychological therapies in a South London CAMHS from 2003-2006. Child and Adolescent Mental Health. 2007;12(2):70-2.

14. Hetrick SE, Thompson A, Yuen K, Finch S, Parker AG. Is there a gap between recommended and 'real world' practice in the management of depression in young people? A medical file audit of practice. BMC Health Services. 2012;12:178. 
15. Hetrick SE, Simmons M, Thompson A, Parker AG. What are specialist mental health clinician attitudes to guideline recommendations for the treatment of depression in young people? Aust N Z J Psychiatry. 2011;45(11):993-1001. 16. Spitzer R, Kroenke K, Williams J. Validation and utility of a self-report version of PRIME-MD: the PHQ Primary Care Study. Journal of the American Medical Association. 1999;282:1737-44.

17. Richardson L, McCauley E, Katon W. Collaborative care for adolescent depression: A pilot study. Gen Hosp Psychiatry. 2008;31:36-45.

18. Chen TM, Huang FY, Chang C, Chung H. Using the PHQ-9 for depression screening and treatment monitoring for Chinese Americans in primary care. Psychiatr Serv. 2006;57:976-81.

19. Patten SB, Schopflocher D. Longitudinal epidemiology of major depression as assessed by the Brief Patient Health Questionnaire (PHQ-9). Compr Psychiatry. 2009;50:26-33.

20. Reynolds WM. Suicidal Ideation Questionnaire (SIQ): Professional Manual. Odessa, FL: Psycholgogical Assessment Resources; 1987.

21. Robinson J, Hetrick SE, Martin C. Preventing suicide in young people: systematic review Australian New Zealand Journal of Psychiatry. 2011;45:3-26. 22. Reynolds WM, Mazza JJ. Assessment of suicidal ideation in inner-city children and young adolescents: reliability and validity of the Suicidal Ideation Questionniare-JR. School Psychology Review. 1999;28(1):17-30.

23. Hetrick SE, Merry S, McKenzie J, Sindahl P, Proctor M. Selective serotonin reuptake inhibitors (SSRIs) for depressive disorders in children and adolescents (Review). Cochrane Database of Systematic Reviews In: The Cochrane Library 2007; Issue 3(Art. No.: CD004851. DOI: 10.1002/14651858). 24. Braun V, Clarke V. Using thematic analysis in psychology. Qualitative Research in Psychology. 2006;3:77-101.

25. Barlow J, Wright C, Sheasby J, Turner A, Hainsworth J. Self-management approaches for people with chronic conditions: A review. Patient Education and Counselling. 2002;48(2):177-87.

26. Hetrick SE, Simmons MB, Sanci L, Gunn J. Monitoring depressive symptoms and suicidality in young people in primary care settings: focus groups with general practitioners. Aust Fam Physician. 2013; In press (accepted 21 November).

27. Timimi S, Tetley D, Burgoine W, Walker G. Outcome Orientated Child and Adolescent Mental Health Services (OO-CAMHS): a whole service model. Clin Child Psychol Psychiatry. 2013;18(2):169-84. Epub 2012/05/03.

28. Timimi S, Tetley D, Burgoine W, Walker G. Outcome Orientated Child and Adolescent Mental Health Services (OO-CAMHS): a whole service model. Clinical Child Psycholology and Psychiatry. 2013;18(2):164-84. 
Reviewer: 1

1. This is an interesting and well written paper reporting on an exploratory study to develop and evaluate an online monitoring tool.

Thank you for your positive and constructive feedback, which we appreciate and in addressing hope that the manuscript is significantly improved. We found it difficult to include all the detail that might have been included in response to these comments because of the word limit of the brief report style. Further, given it is a small pilot study, some comments could not be specifically addressed except to ensure that we have outlined the limitations and pointed to the need for further study. We are currently testing the tool in a larger sample and will publish several papers on the basis of this study and wish to be able to cite a published article about its development and pilot testing; as such if published in this journal, the paper will be well cited.

Other text changes have been made in order to accommodate the changes made in response to reviewers within the word limit.

2. All participants were in a program for those with moderate to severe depression. How is this determination of severity currently made? Please report also whether all of the participants in the study were on medication. It would also be useful to provide a brief description of the sort of treatment participating clients were receiving at this service.

We have included the severity ratings based on baseline $P H Q-9$ scores and the number who were on medication in the results. We have include a description of the treatment young people receive in the YMC in the methods section.

3. Please provide the age range and mean age for clients. Also provide a suggestion as to the age range that the tool is suitable for. As a comment, I am also interested in whether younger clients (around 15 years) may respond differently to the tool in comparison to those clients aged around 24 years of age.

YMC is part of Orygen Youth Health, a tertiary or specialist youth mental health service, that treats about 150 patients between the ages of 15 and 24 each year, 85\% of whom have a diagnosis of major depressive disorder. Given the setting and the number of young people who can be seen each year, it is only those who have more severe disorder who are able to be accepted into the clinic. The PHQ is the tool that the clinic already used to assess depression severity, along clinical assessment. One of the issues we identified in an earlier study is the lack of routine and systematic assessment of the severity of depression on entry to the clinic(1), and in part the tool aims to address this (given this is a brief report we have been limited in the detail we can provide). Using the PHQ scores generated from completion of the tool, the mean PHQ scores indicated $X$ severity of depression. Patients are reviewed regularly by the psychiatrists, and therapists deliver weekly CBT and case management. All patients have access to after- 
hours crisis care and inpatient treatment when needed. During their course of treatment $80 \%$ of the patients will be treated with medication; in this study all young people were on medication. The median length of treatment is 6 months.

Much of this detail has been added, although given the word limit it was not possible to give in depth description as above.

The tools employed in the monitoring tool are those that have been already validated and are widely used in young people as young as $11(2,3)$ or $12(4)$. In this study the mean age of participants was 18.74 (SD = 2.65). We have added the mean age into the results section. In this study we did not investigate how age modified the way in which the tool was completed; but this is a question that could and should be addressed in future studies and a comment to this end has been included in the paper.

4. Please provide some further information as to how clinicians received information from the survey. It is stated they receive a chart of the client's PHQ-9 and SIQ-JR after 4 weeks, but do they have access to the raw data before this time. It is stated that the suicide items are completed in the presence of the clinician so they have immediate access to that. However, it is unclear how the clinicians receive the information from the survey. For example, how do they know if the client has completed the survey, how quickly can they access the information, what format is data provided in, how do they feed back this information to clients, and how quickly is this feedback provided? In addition, how is the data from external locations with Internet access obtained?

We agree more detail is required here. We had outlined that the tool was designed to be online and was available to be completed on iPads within the YMC (outlined in 'Phase 1: Development' section); however, in the procedure section have reiterated that the suicide items were completed on the iPad and added a description about how clinicians then received feedback (These were completed at the beginning of the clients regular treatment session with their clinician on an iPad. Once finished the young person handed the iPad to their clinician, with the iPad programmed to display responses to the SIQ-JR with an indication regarding the meaning of the scores (i.e. SIQ-JR score of $>31$ or a score of $>4$ on items $2,3,4,7,8,9,13$ or 18). We have then included a statement to highlight that clinicians did not have access to raw scores. We were hoping to test the impact of receiving feedback in graphs by delaying access to this, however, due to the small numbers included could not examine this in a meaningful way.

5. The use of technology, especially with young people, can be very helpful as an adjunct, or in some cases, stand-alone treatment. I think further suggestions could be given in the paper as to how to maximise the utility of the tool for both clients and clinicians. For example:

- If clients complete the form remotely, what feedback do they get immediately and what does happen if they are reporting high levels of symptoms or serious side 
effects to medications?;

- If there are medication side effects, who responds to those concerns and how;

- When does their clinician respond to their survey data?;

- How can the clinician best use the data provided and how do they discuss this with the client?;

- How will the risk assessment be managed and will this always be conducted in person?

While we agree these are good suggestions, we think this is probably beyond the scope of the current study and its results; and importantly difficult to include given the word length. As mentioned below we did not have ethics approval to examine use of feedback to clients independent of clinicians so we cannot make any suggestions in this regard. We have added to the paper that clinicians received an email about side effects that were endorsed by young people. Clinicians were seeing these clients regularly with standard clinical assessment of depression severity taking place at each treatment. We have included in the paper more detail about the way clinicians received feedback about suicidal ideation/risk and that they responded as necessary in that session. We have tried (within the word limit) to include a little more detail in the discussion about the potential usefulness of the clinician sharing scores with the client.

6. If this tool is to be used more broadly, then there will be many young people who will not be on antidepressant medication so I assume that part of the checklist would be left blank.

Yes, that is correct. Programming can ensure that this section is turned off or on. We have indicated in the discussion that it may be useful to investigate how those on medication use the tool.

7. It is also stated that no clinicians showed their clients graphs of their data. Can you comment on the reasons for this? In terms of therapeutic process, I think it is essential that clients are provided with graphs or other useful visual aids about their own symptoms on which they are reporting. This helps build therapeutic alliance and also encourages clients to play a more active role in their own health care. This could also build mental health literacy and perhaps assist clients to identify more quickly when there may be a decline in functioning and perhaps learn some strategies to address this. Can you also discuss how the feedback to clients can be built into this tool?

This was intriguing to us as we agree, it could be very useful and as we reported in our results, young people were keen to see their scores. In a larger study we are currently undertaking we have put in place mechanisms whereby the graphs are available each time the tool is completed and are available to clients. This is still via their clinicians because as a research tool, the ethics committee who reviewed this were very strongly against the idea of young people have access to their scores if not in the presence of 
their clinician who has a role in ensuring this information is interpreted correctly; and indeed that it becomes part of the therapeutic process. As such therefore, we are still relying on clinicians to judge whether they believe it is useful and helpful to show their clients the graphs. We have added some detail about the need to investigate feedback to clients.

8. Please comment on how this tool may be used in different services, particularly those that may already have some protocols in place.

We have reported that one of the key findings is the need to ensure the tool is embedded into existing practice - we have slightly altered the wording to highlight that this would need to be individualized to the different types of practices that the tool might be used in to ensure it fits with existing protocols. We have referred specifically to a paper that is currently in press about how the tool could be used in general practice where we also highlight one of the key findings from this paper about how the tool needs to be appropriately incorporated into these types of services.

9. While I fully support the idea of regular monitoring of symptoms and medications, I am not sure whether a monitoring tool such as the one developed in the current study will address the issue from the research findings that regular monitoring is not uniformly implemented. It is also known from research that clinicians who monitor clients' progress and their own performance, achieve better therapy outcomes. It is concerning therefore, that symptoms of depression and response to treatment are not regularly reviewed, given they are key indicators of progress and treatment effectiveness. Further given the increased risk for suicidal ideation and intent associated with depression, it is also necessary to undertake some type of regular check with clients to assess their ongoing status. Given there are quite brief screening measures that already exist, I am not sure that it is the lack of an evaluation tool that is the key issue here.

We agree and this pilot study was unable to test whether the tool could improve adherence to guideline recommendations. We have included more discussion of issues relevant to the successful implementation of the tool in regards to its use improving adherence to guideline recommendations, including citing an example of a study that shows regular review of response to treatment improves clinical outcome; and importantly stated that the tools effectiveness in improving guideline concordant practice requires further testing.

10. It is noted that there were no doctors involved in the study. Please comment on why this was the case and also on the what qualifications case managers held.

We have added a note regarding the qualifications of the case managers and why doctors did not participate. 
11. As a further suggestion, it would be useful if you could introduce this evaluation tool into your service as a larger trial and also collect brief satisfaction data from the clients (this could be a simple tick box format at the end of the survey asking about the usefulness of the survey, how difficult they found it to complete, and likelihood to continuing using the tool). Additional focus groups/interviews with clinicians could examine implementation of the tool, barriers/difficulties, and impact on treatment. This would make a very good addition to the current paper and should allow for some clearer suggestions to be made about the tool and its delivery.

We have received funding to implement the tool, refined on the basis of this pilot study, into this service, a headspace (enhanced primary care) and general practice and have included collection of data with regard to satisfaction. This study is underway and results will be available next year. We will publish the results of this study in separate papers, given the tool is slightly different and that we have taken on board the findings of this study (which are confirmed by this reviewer) with regards to the implementation of the tool across a range of services. We think it is important to publish data on the development and pilot study of the first version of the tool to provide some context to its ongoing development and implementation into practice.

\section{Reviewer: 2}

1. The paper describes a first step towards the design of an etool for assessment of depression. At this stage, it is limited to programming of existing instruments, and requires web access, and has a substantial number of questions for repeated monitoring. Adherence to monitoring appears limited as yet, and acceptability also has room of significant improvement.

Development (or adaptation) of a shorter yet valid and sensitive instrument--ideally programmed for use on both mobile phones an iPads, and in an app format that could be used when web connections are unavailable--would be a significant contribution to the field. The current data could be reported in a paper that describes further developments along these lines.

We agree that more development is required, including further testing and have in mind that the end result could be connectivity to such a tool via a mobile phone app. Given the innovation embedded in this program of research we think it is important to publish data on the development of the tool and the learning's from this pilot study given others may well be developing similar tools. We are currently testing a version of this tool, modified on the basis of this pilot study, in a larger sample and hope to publish findings in 2015.

1. Hetrick SE, Thompson A, Yuen K, Finch S, Parker AG. Is there a gap between recommended and 'real world' practice in the management of depression in young people? A medical file audit of practice. BMC Health Services. 2012;12:178. 
2. Reynolds WM, Mazza JJ. Assessment of suicidal ideation in inner-city children and young adolescents: reliability and validity of the Suicidal Ideation Questionniare-JR. School Psychology Review. 1999;28(1):17-30.

3. Reynolds WM. Suicidal Ideation Questionnaire (SIQ): Professional Manual. Odessa, FL: Psycholgogical Assessment Resources; 1987.

4. Spitzer R, Kroenke K, Williams J. Validation and utility of a self-report version of PRIME-MD: the PHQ Primary Care Study. Journal of the American Medical Association. 1999;282:1737-44. 


\section{University Library}

\section{- M M N E R VA A gateway to Melbourne's research publications}

Minerva Access is the Institutional Repository of The University of Melbourne

Author/s:

Hetrick, SE;Dellosa, MK;Simmons, MB;Phillips, L

Title:

Development and pilot testing of an online monitoring tool of depression symptoms and side effects for young people being treated for depression

Date:

2015-02-01

\section{Citation:}

Hetrick, S. E., Dellosa, M. K., Simmons, M. B. \& Phillips, L. (2015). Development and pilot testing of an online monitoring tool of depression symptoms and side effects for young people being treated for depression. EARLY INTERVENTION IN PSYCHIATRY, 9 (1), pp.66-69. https://doi.org/10.1111/eip.12127.

Persistent Link:

http://hdl.handle.net/11343/59209 